\title{
Logical Intervention in the Form of Work Breakdown Strategy using Object Constraint Language for e-Commerce Application
}

\author{
Shikha Singh ${ }^{1}$, Dr.Manuj Darbari ${ }^{2}$ \\ Department of Computer Science and Engineering \\ School of Engineering \\ Babu Banarasi Das University \\ Lucknow (Uttar Pradesh), INDIA
}

\begin{abstract}
This paper proposes a framework for Rule Based inhibition on e-commerce website for prevention of double payment and computing time invariant for concurrent event handling. Authors have analyzed computational models in terms of Customer segmentation replicating their buying characteristics and Dialogue level constraint establishment through OCL. The tool used are MDT-OCL and matching logic for logic level interpretation. The MDT tool generates Context Syntax Tree. We have used LPG grammar to be applied WBS codification by differentiating Descriptive Noun containing description about the products and associated verbs about the product. Authors have used Eclipse plug-ins to embed it logic constraint mapping to check for any ontological errors and double selection and payment errors.
\end{abstract}

Keywords-OCL; e-commerce; concurrent handling; work breakdown structure; augmented querying

\section{INTRODUCTION}

This Nowadays there is change in trend from physical movement of client to supplier's location and finally purchasing a product, its digital millennium e-commerce website has gained considerable importance. There are various categorization of e-commerce i.e.B2B, B2C \& C\&B. The basic idea is to provide ease of use to the client be it any category of client. In all the e-commerce applications main focus is on processing delay and high throughput in terms of outcome as desired by the user. Object constraint language was used in many earlier literatures but failed to achieve high throughput as processing time was significantly high. Chomicki [1] proposes a efficient method on semantic interconnections. Barbara [7] also suggested efficient method for component based modeling for e-commerce website.

All the above proposals have some drawback or the other; for example a client way want to be guided to right product, but unfortunately the time as well as the outcome of the query are main cause of concern. Proper merging from the various databases is a big problem.

This paper proposes a method to enhance the linking of various databases, by forming clusters thereby increasing the throughput of the query. Secondly as compared to normal queries Argumentative Query provides much increased output using OCL logic foundations. By embedding theoretical formal syntax providing an Argumentative logic to reach to a final efficient query outcome.

The paper starts with a brief introduction discussing about the current problems in e-commerce website, followed by exhaustive literature survey on OCL and its application in ecommerce. The third section proposed solution taking the case study of Amazon website. The proposed solution is supported by Analysis showing how augmentative constraint language is related with satisfaction level and response time. Lastly the paper is supported by conclusion and future scope of the work.

\section{LITERATURE REVIEW}

Author in [2] emphasis on use of fuzzy sets and possibility theory as a realistic approach for the representation of various categories of constraints. Author in [3] represents how transaction concepts apply to distributed systems and how to build high performance, high availability applications with finite budgets and risks. Author in [4] discusses a systematic approach towards preference engineering, preferences as soft constraints and a query model, along with its algebra and decomposition. Deductive arguments and counterarguments were been discussed with formalizing them using logical languages [5]. Author in [6] discussed the transformation of Unified Modeling Language (UML) models into software execution models and simulate the performance prediction for an e-commerce application. Author in [8] propose a web perusing history mining based client inclination revelation technique for web based business frameworks. Another strategy called UPSAWBH (User Preference Similarity Calculation Algorithm Based on Web Browsing History), which measure the degree of clients' inclination likeness based on their website page click designs, is advanced. Author in [9] presented, a preference update model to address the problem which arises, when customers issue requests based on out of date information in e-commerce application systems. They also proposed a group evaluation strategy for preference update processing in multi-database environment. Approaches for controlling the social actions that web 2.0 applications allow users to execute [10]. A method to enhance the accuracy of the model by adding static semantics to the SMP2 Meta model is proposed [11]. The control over these actions is defined with UML/Object Constraint Language (OCL) and then demonstrated through a prototype system. Verbalization of 
business rules deciphers the principles communicated in a design language into semi-regular articulations. This permits business specialists to approve models communicated in a plan language without suggesting any aptitude on this language [12]. A change instrument is proposed to robotize verbalization and applied to OCL (Object Constraint Language) limitations in the utility area. A structure to help formal displaying and agreements for information driven web administrations is show to be utilized as to confirm accuracy properties for synthesis of services [13]. OCLLib, OCLUnit and OCLDoc are proposed in [14]. OCL lip makes easier for the development of OCL expressions and constraints. It makes a high reuse factor which is configurable and testable named as OCL unit and OCL DOC. A security policy using Object Constraint Language has been developed in [15] secure MOVA tool utilized to answer the result of the proposed approval verification of non-trivial security properties. An access control Meta model has been developed [16] using unified modeling language (UML). This modeling language is independent of access control requirement using genetic mutation along with independentness from specific implementations. An experiments framework has been proposed in [17], for evaluation the constraints in object constraints language, along with Automated support for OCL refactoring. Modeling Business process is one of the important issues in maintaining competitions and dealing with challenges in business environment. Model driven software engineering is the new paradigm for the designing software. For meta modeling, UML has been used to model the problem along with a static semantic of the language specified by the object constraint language (OCL) as discussed in detail in [18]. In [19] temporal and history based authorization constraints are represented by the OCL. Also, first orders linear temporal logic has been utilized for formally satisfy the constraints. OCL is playing an important role in object oriented software development in the framework of UML and Eclipse modeling framework (ENF). In [20] an extension of OCL, Soil (Simple OCL-like Imperative Language) has been proposed, using declarative representation. Author in [21] presents a UML composition formalization that strictly adheres to the UML specification. This formalization paves the way for future work to check the consistency between a design model and its implementation in terms of the UML composition. Author in [22] presents a combined Object Constraint Language (OCL) and Object Role Model (ORM) for integrity constraints modeling, and demonstrates an implementation which enforces them by using a commercially available DBMS. Generally, OCL constraints are written manually, which may cause incorrectness and extra overhead. Therefore, generating OCL constraints template for UML models is a superior solution and is presented in [23]. The OCL constraints template automatically generated can be used as a reference for software designers. Author in [24] makes an investigation into the recent developments and explores the role of OCL in the current scenario and its future applications. Author in [25] presented an ontology-based approach for verification of business processes. They specified business rules as a logic program and used ontology reasoner for discovering model elements which violate the rules. Author in [26] exhibited ontology-based data intensive EIS (Enterprise Information System), which is notification- oriented. Author in
[27] proposed domain specific language called ReSA for an embedded system. The ReSA utilizes axioms of ontology for specification of the embedded system. They perform scalable formal verification of various Simulink models. Sunitha, E. V. et al. 2018 [28], UML is achieved by using hundred percentage automation in code technology system fashions will make a drastic development in software program enterprise. This model examines a way to enhance the code age from UML models, with the assistance of "OCL". It likewise investigates the potential outcomes to combine OCL in UML action models and produce code from the OCL unrivaled movement diagrams. Hammad, Muhammad et al. 2017 [29], The "OCL" is generally utilized intended for identifying an extra restriction on representation. To assist practitioners as well as researchers to indicate "OCL" restriction, they intended and advanced an Internet-based device referred to as interactive OCL (iOCL) for interactively identify a restriction on a given version. The middle concept behind iOCL is to here and show only appropriate information (e.G., operations) of "OCL" to customers at a specified pace of restriction requirement procedure, further toward assisting modelers with its syntax. Thus, they finish that iOCL can assist the system of "OCL" restriction requirement. Zaragoza, Mechelle Grace et al. 2018 [30], The method explains software of cellular integration additives in public organization and e-commerce as a software improvement technique to basically incorporate the specific primary mechanism of the era right into a solitary net-primarily based explanation. They tested a systematic improvement process for the software agent using additives and UML. They initially organized the agent factor arrangement and form it. Based on this, we evolved a cellular application for social commercial enterprise packages. They incorporate the modulebased software program system into Drupal's content control system. Author in [31] proposed the utilization of security presentation flexibility expectation working method.

\section{PROPOSED SOLUTION}

This paper proposes a constraint language in e-commerce application with emphasis on cognitive search and Behavioral transposition of customers during buying process. Various computational models are identified and analyzed and is discussed in Table I.

TABLE I. A COMPARISON OF VARIOUS COMPUTATIONAL MODELS

\begin{tabular}{|c|c|c|c|c|}
\hline & \multicolumn{4}{|l|}{ LEVELS } \\
\hline Features & $\begin{array}{l}\text { Dialectica } \\
1\end{array}$ & Logical & Dialogue & Rhetorical \\
\hline Argument & $\begin{array}{l}\text { Which } \\
\text { Argument } \\
\text { wins }\end{array}$ & $\begin{array}{l}\text { Arguments are } \\
\text { Atomic in } \\
\text { Nature }\end{array}$ & $\begin{array}{l}\text { Agents } \\
\text { Exchange } \\
\text { Arguments in } \\
\text { Various } \\
\text { Activities }\end{array}$ & $\begin{array}{l}\text { Aim of } \\
\text { Argument } \\
\text { is wider }\end{array}$ \\
\hline $\begin{array}{l}\text { Methodolog } \\
\text { y }\end{array}$ & $\begin{array}{l}\text { Argument } \\
\text { s \& } \\
\text { Counter } \\
\text { Argument } \\
\text { s }\end{array}$ & $\begin{array}{l}\text { Arguments } \\
\text { taken from } \\
\text { Knowledgebas } \\
\text { e }\end{array}$ & $\begin{array}{l}\text { Dialogue } \\
\text { games are } \\
\text { created in the } \\
\text { form of } \\
\text { Communicativ } \\
\text { e Acts }\end{array}$ & $\begin{array}{l}\text { By way of } \\
\text { Persuasio } \\
\text { n using } \\
\text { Threats } \\
\text { and } \\
\text { Rewards }\end{array}$ \\
\hline
\end{tabular}


In order to apply these computational model to check the buying behavior we first develop the logical foundation of Rules base as suggested by E. Franconic et al.

$$
\begin{aligned}
& \text { OCL: Set:: =OCL - Set } \longrightarrow \text { Union (OCL - set) } \\
& \mathrm{OCL} \text { - Set } \longrightarrow \text { elect (Var |OCL - Bool) | } \\
& \mathrm{OCL} \text { - Set } \longrightarrow \text { reject (Var } \mid \mathrm{OCL} \text { - Bool) | } \\
& \text { Class.allinstances }
\end{aligned}
$$

The main issue with the above problem is application of constraints in processing their query with large amount of concurrent request to be using the limited resource. This happens with the case of sale announced by various companies during peak and off peak season. Here comes the role of computational model using Argumentative models and work Breakdown strategy to provide better customer satisfaction and prevention of chocking at the server end. Consider an ecommerce site where a customer wants to buy a wrist watch and there is another query which is related to stainless steel watch strap only, then both queries will have the same resources to be shared we can apply Argumentative models for evaluating the customer's request (Fig. 1).

OCL logical Rule Base has been formed like in this case it can be written as:

Context wrist watch inv Watches:

self.purchase $=$ 'any'implies.self. watch $\longrightarrow$ steelstrap.

Can be satisfied using a mix of preference model which will discriminate the customer based on the priority and its relationship with the resource availability i.e. by way of Argumentative Rules applied with OCL to form the Work breakdown structure (WBS).
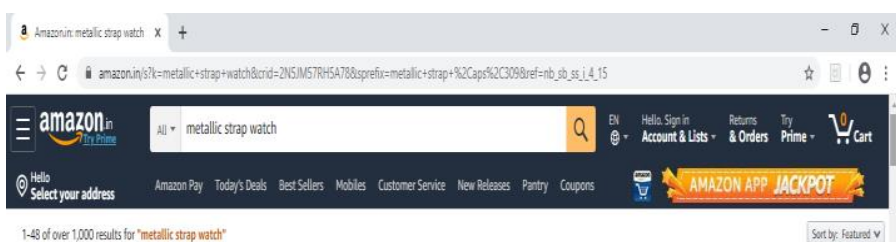

1.48 o f ove 1,000 results for 'metallic stap watd"

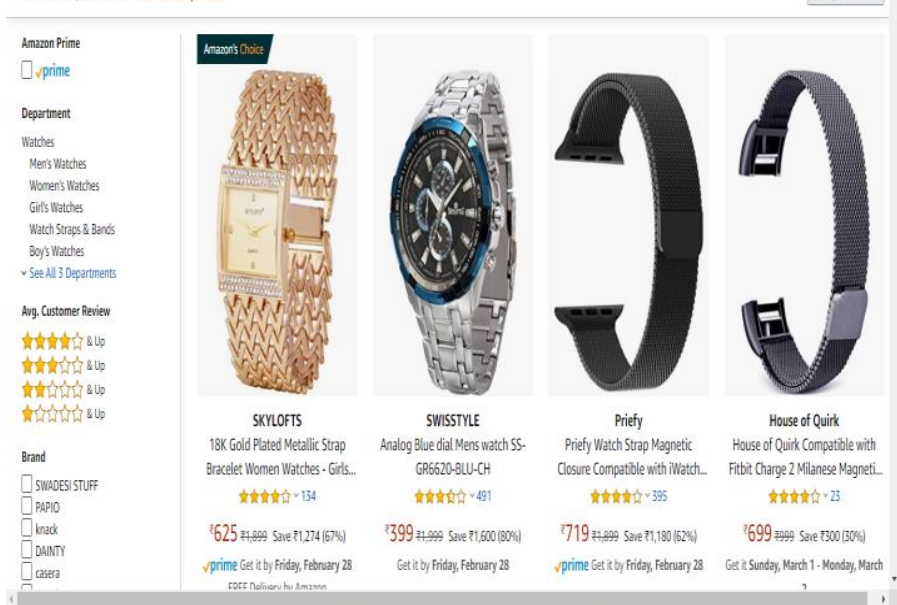

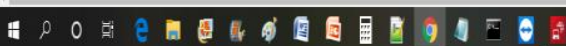

Fig 1. Snapshot of wrist watches purchase.

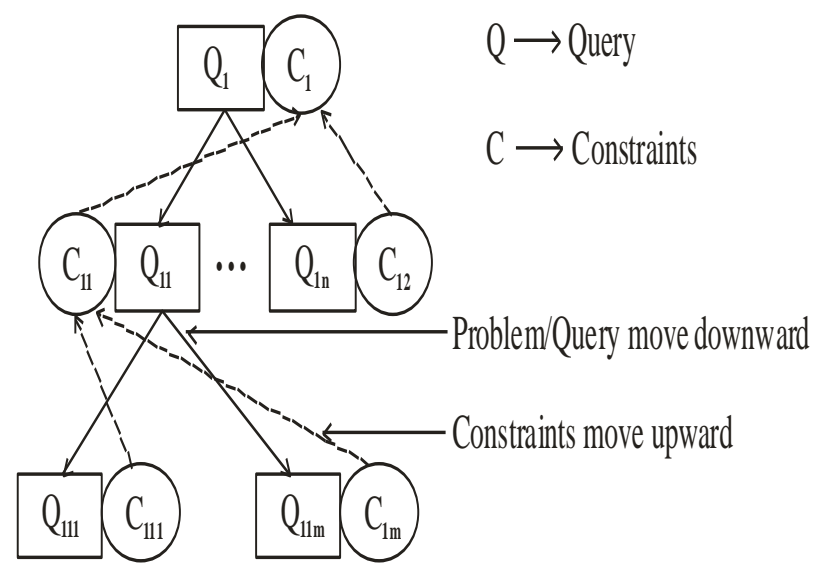

Fig 2. Task Reduction being achieved with Query and Constraints (OCL).

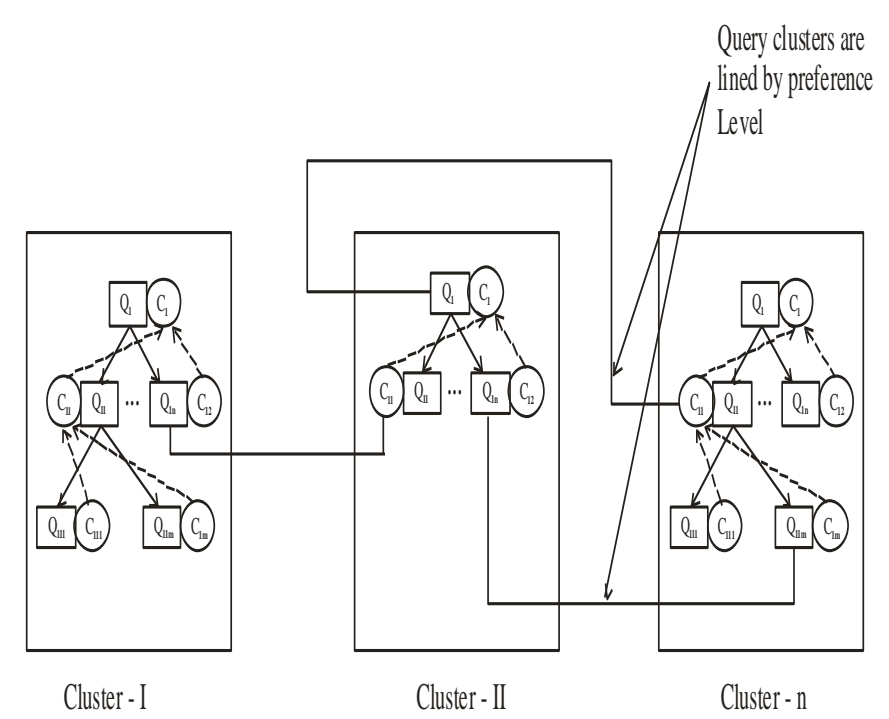

Fig 3. Cluster formation of Queries with OCL Logic.

The above Fig. 2 represents the Task Reduction technique as suggested by (Michanel \&Bourdan, 2007). This is combined with Argument Centric preference Adaptation Model with OCL Logic constraints at each and every layer.

The basic idea of this model is to break down the searching item query by creating a cluster and then applying the cluster constraints to solve the problem.

These clusters in Fig. 3 represent the particular group with concurrent request of resources.

Let ' $\mathrm{Q}$ ' be the instance which is a result of two instances Q1 and $\mathrm{Q} 2$ requesting the particular resources.

Now according to argumentativeness the constraint should be applied on the Model which can be written as

Q. allInstances ( ) $\longrightarrow$ for All $\mathrm{r} \mid$ Q1.allInstances ( ) $\rightarrow$ xists (cluster1 | cluster2| cluster $\mathrm{n}$ )

Equation - I 


\section{A. The decision Making process using Argumentative} Constraints

Set 'Q' be the query with ' $\mathrm{S}$ ' as the solution derived in the form of equation-I, above, we write the rules using temporal logic in Object constraint logic as argumentative graph:

$\mathrm{Q}_{1}=\left\{\mathrm{S}_{1} \mathrm{OK}\right.$ (Constraint set 1$), \mathrm{S}_{3} \wedge$ (Constraint set 3$\left.) \rightarrow \mathrm{S}_{1} \wedge \neg \mathrm{S}_{3}\right\}$

$\mathrm{Q}_{2}=\left\{\mathrm{S}_{4} \mathrm{OK}(\right.$ Constraint set 4$), \mathrm{S}_{3} \wedge$ (Constraint set 3$\left.) \rightarrow \mathrm{S}_{4} \wedge \neg \mathrm{S}_{3}\right\}$

$\mathrm{Q}_{\mathrm{n}}=\left\{\mathrm{S}_{\mathrm{m}} \mathrm{OK}(\right.$ Constraint set $\mathrm{m}), \mathrm{S}_{\mathrm{k}-1} \wedge$ (Constraint $\mathrm{k}-1) \rightarrow \mathrm{S}_{\mathrm{m}}^{\wedge} \neg \mathrm{S}_{\mathrm{k}-1}$

Where:

Q1 =Watches

Q2 = Metallic Watches

Q3 = Metallic Strap Watches Dial

Q4 = Watch with Metallic$$
\text { S4 = Best choice is Fitbit }
$$$$
\mathrm{S} 1=\text { Best choice is }
$$$$
\text { Garmin }
$$

$$
\mathrm{S} 2=\text { Best choice is Apple }
$$$$
\mathrm{S} 3=\text { Best choice is }
$$$$
\text { S5 = Garmin with }
$$$$
\text { Metallic Watch }
$$$$
\text { Watch }
$$$$
\text { S6 = Apple with Metallic }
$$

\section{Dial}

S7 = Apple with Metallic

The above constraint of Object Argumentative solution is derived by the help of dialogue rule clusters arranged in Work Breakdown manner where the Queries moves downward and constraint move upward to narrow down the search and also avoid concurrent processing.

Each cluster can be represented as presented in Fig. 4.

\begin{tabular}{|c|c|c|c|}
\hline $\begin{array}{l}\text { Layer } \\
\text { Number }\end{array}$ & $\begin{array}{c}\text { Flow of } \\
\text { Query }\end{array}$ & $\begin{array}{l}\text { Constraints } \\
\text { Used }\end{array}$ & $\begin{array}{l}\text { Solution } \\
\text { Derived }\end{array}$ \\
\hline 1 & $\left\{Q_{1}\right\}-$ & $\rightarrow\left\{C_{3}\right\}$ & $\rightarrow \mathrm{S}_{1}$ \\
\hline 2 & $\left\{Q_{3}\right\}$ & $\rightarrow\left\{C_{2}\right\}$ & $\mathrm{S}_{3}$ \\
\hline 3 & $\left\{Q_{4}\right\}$ & $\rightarrow\left\{\mathrm{C}_{5}\right\}$ & $\mathrm{S}_{2}$ \\
\hline 4 & $\left\{Q_{5}\right\}$ & $\rightarrow\left\{C_{4}\right\}$ & $\mathrm{S}_{4}$ \\
\hline 5 & $\left\{\mathrm{Q}_{2}\right\}$ & $\rightarrow\left\{\mathrm{C}_{1}\right\}$ & $\mathrm{S}_{5}$ \\
\hline
\end{tabular}

Fig 4. Block Inside representation of cluster.

We are able to derive the Flow of information with constraint to check two factors:

(i). One is Concurrency of the Request (ii). Second is Ease of use to the user with Argumentative OCL at the backend

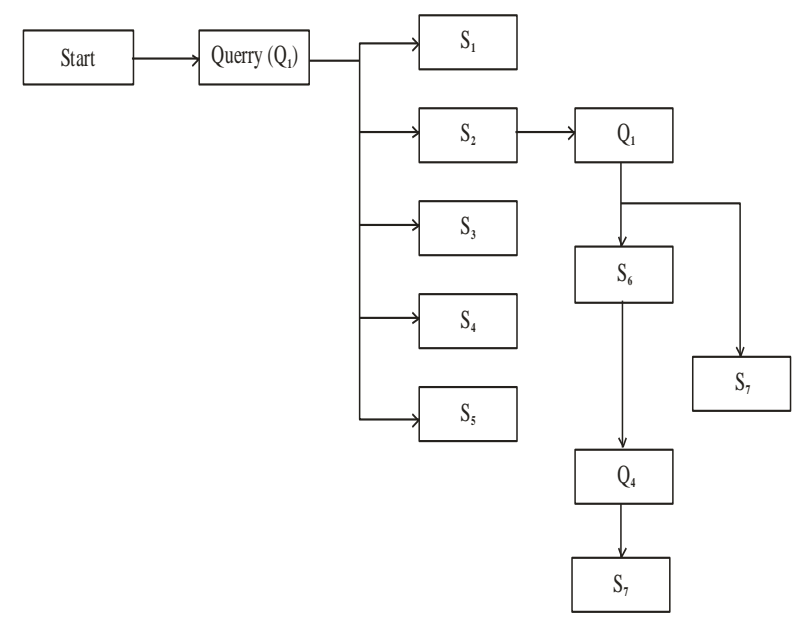

Fig 5. Argumentative OCL has been used to derive the solution $\mathrm{S}_{7}$.

The above WBS protocol constraint shows the breakdown of Query set using Rhetorical Level constraint forcing the Client Query to leave $S_{6}$ Resource and move to $S_{7}$ solution, which is the derived solution set discussed in Fig. 5.

\section{B. Analysis}

Based on the Online purchase of Metallic watch, we represent the success rate of our model for standard query resulting into the desired output with a mean of three Query Request per hour we are to plot the success rate and Query processing time using Argumentative constraint Language as shown in Fig. 6 and Fig. 7, respectively.

The above two comparative figures shows the graph of satisfaction level and response time using MySQL normal Query and Argumentative OCL embedded with MySQL Query interface. Number of queries was generated and regards to the watch purchase and Argumentative Query Language showed much efficient output in terms of Response time and Satisfaction Level as the right output.

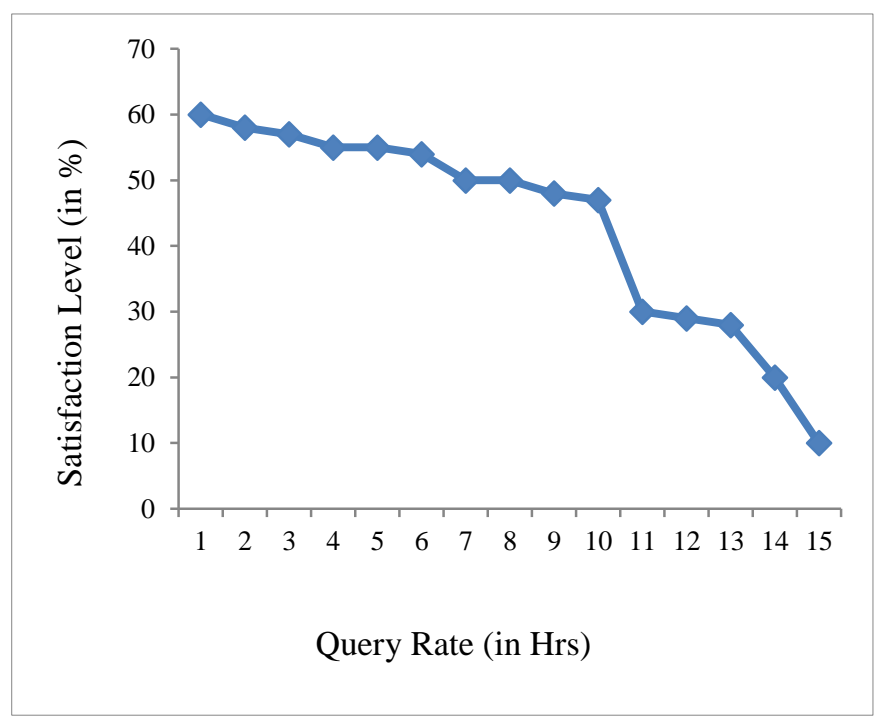

Fig 6. Success Rate of Argumentative Constraint Language. 


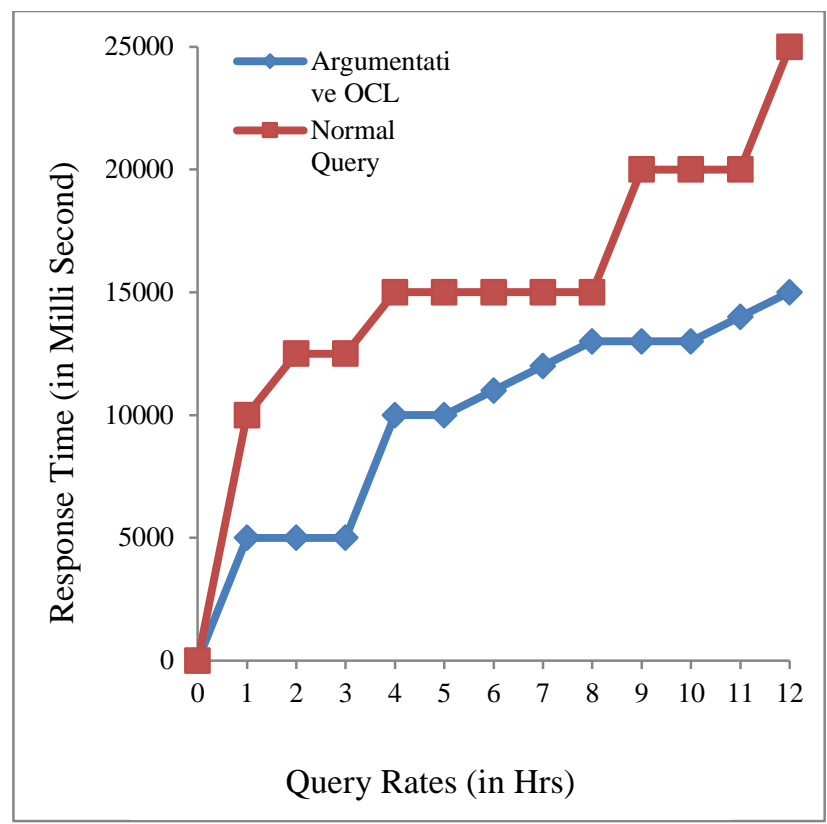

Fig 7. Comparison of Response Time.

\section{CONCLUSION AND FUTURE SCOPE}

The paper discusses the issue of application of constraints on Query search for a particular product, and then the satisfaction level in terms of the right matches is analyzed. The paper proposes the up gradation of normal OCL by using Argumentative computational model thereby enhancing the capability of search in terms of more persuasion and directional.

The paper suggests how augmentative computational method provides a more formalized and refined method of querying. The problem of time of query is simplified and satisfaction level has significantly increased along with drop in response time with respect to normal query. It was achieved by using cluster in which various sets of work breakdown Ontologies are stored and are retrieved used argumentative centric preference adaption using OCL.

Future work relates to developing a parser where we can directly embedded the rules of argumentative search and it can retrieve from it.

\section{ACKNOWLEDGMENTS}

My Sincere Thanks to my Guide and mentor Dr. Manuj Darbari Sir without whom this work would not have been possible.

\section{REFERENCES}

[1] Chomicki, J. (2019) Preference formulas in relational queries, ACM Transaction of Database Systems.

[2] Dubois, D. (1996) Possibility theory in constraint satisfaction problems: Handling priority, preference and uncertainity. Applied Intelligence, 6, 287-309.

[3] Gray, J \& Reuter, A.(1993) Transaction Processing : Concept and Techniques, Morgan Kaufmann, Sam Mateo, C.A.

[4] Kiessling, W. Foundatin of Preferences in Database Systems. Proc of 2002 IEEE Int. Conf. on Data Mining (ICDM '02).

[5] Cialdini, R : Influence : The Psychology of Persuasion, Harper Cellins (1984).
[6] Besnard, P., Hunter, A : Constructing Arguments graphs with deductive Arguments : A tutorial Argument \& Computation 5(1), 5-30 (2014).

[7] Barbara and Massimo, Designing components for e-service, Proceedings of VLDB workshop on Technologies for e-services, Cairo, Egypt, 2000.

[8] D Evange Geetha, Ch Ram Mohan Reddy, T V Suresh Kumar, K Rajani Kanth, "Performance Modeling and Evaluation of e-commerce Systems Using UML 2.0", proc of $8^{\text {th }}$ IEEE, ACIS International Conference on Software Engineering, Artificial Intelligence, Networking and Parallel/Distributed Computing (2007).

[9] P.Li, M.Tu, Z.Xia, "Preference update for e-commerce applications: Model, language, and processing", Springer Science + Business Media LLC 2007, Electronic Commerce Res(2007)7: 17-44.

[10] Z.Maamar, V.Buregio, N.Faci, D.Benslimane, Q.Z.Sheng, "Controlling "Web 2.0 applications in the workplace,19th International Enterprise Distributed Object Computing Conference(EDOC), pp.191-200, 2015.

[11] L.Haibing, Z.Ning ,L.Yonglin,L.Xiaobo ,Z.Yifan "EMF Based Validation Methods of the Static Semantics of Models", 2nd International conference on Information Science and Control engineering, IEEE, pp. 207-211, 2015.

[12] R.Baghli, B.Traverson "Verbalization of business Rules Application to OCL Constraints in the Utility Domain", 2nd International conference on Model Driven engineering and software development, IEEE, pp. 348-355, 2014.

[13] I.Saleh, G.Kulczycki, M.B.Blake, "Formal Specification and Verification of Data Centric Service Composition", International conference on Web Services (ICWS) IEEE, pp. 131-138, 2010.

[14] C.-O. Joanna, OCLLib, OCLUnit, OCLDoc: pragmatic extensions for the object constraints language, LNCS vol. 5795, 2009, 665-669.

[15] Basin, M. Clavel, J. Doser, M. Eaea, automated analysis of security design models intonation and software technology, vol. 51 no. 5, 2009,pp 815-831.

[16] S. Nadera, K. Hemanth, A. Kamel, L. Luegi, VACML: unified access control modeling language, 2011 4th IFIP international conference on new technologies, modeling and security (NTMS), Canada, pp.1-8.

[17] A.L. Correa, C.M.L. Werner, M. Barros, Refactoring to improve the under standability of specifications written in object constraint language, IET, software, vol. 2, pp-69-90.

[18] C. amelunxen, A. Schurr formalizing model transformation rules for UML/MOF2,IET software, vol. 2 no 3,(2008).pp. 204-222.

[19] K. Sohr, M. Drouineaud, G.J. ahn, M. Gogolla Analyzing and managing role based access control polices, IEEE transactions on knowledge and data engineering, vol. 20(2008),pp. 924-939.

[20] F. Buttner, M. Gogolla, Modular embedding of the object constraints language into a programming language, LNCS, vol.7021, 2011 pp. 124139.

[21] H.M. Chavez, W. Shen , "Formalization of UML Composition in OCL", 11th IEEE/ACIS International Conference on Computer and Information Science. 2012.

[22] J. Saetent, N. Vejkanchana, S.Chittayasothorn, "A Thin Client Application Development using OCL and Conceptual Schema", $6^{\text {th }}$ IEEE International Conference on Internet Technologies and Secured Transactions, Abu Dhabi, UAE, December 2011.

[23] Li Tan, Z. Yangand, J. Xie, "OCL Constraints Automatic Generation for UML Class Diagram”, IEEE 2010

[24] Er. Shivani Gupta, Dr. Dhavleesh Rattan, "Research Paper on Implementation of OCL Constraints in JAVA" International Journal of Advanced Research in Computer Science, ISSN No. 0976-5697,Vol -8, June-2011.

[25] Corea, C., and Delfmann, P., "Detecting Compliance with Business Rules in Ontology-Based Process Modeling", Proceedings of 13th Internationale Tagung Wirtschaftsinformatik, pp. 226-240, 2017.

[26] Liao, Y., Panetto, H., Simão, J.M., and Stadzisz, P.C., "OntologyBased Model-Driven Patterns for Notification-Oriented DataIntensive Enterprise Information Systems", Proceedings of 7th International Conference Information Social Technology, pp. 148-153, 2017. 
[27] Mahmud, N., "Ontology-Based Analysis and Scalable Model Checking of Embedded Systems Models" M\{ä\}lardalen University, 2017

[28] Sunitha, E. V., and Philip Samuel, "Object constraint language for code generation from activity models", Information and Software Technology 103 (2018): 92-111.

[29] Hammad, Muhammad, Tao Yue, Shuai Wang, Shaukat Ali, and Jan F. Nygård. "iOCL: an interactive tool for specifying, validating and evaluating OCL constraints." The science of Computer Programming, Vol. 149 (2017), pp.3-8.
[30] Zaragoza, Mechelle Grace, Haeng-Kon Kim, and YounKy Chung. "Components of Mobile Integration in Social Business and E-commerce Application." In International Conference on Computational Science/Intelligence \& Applied Informatics, pp. 59-68. Springer, Cham, 2018.

[31] Pathak, Nitish, B. M. Singh, and Girish Sharma. "UML 2.0 based framework for the development of secure web application." International Journal of Information Technology 9, no. 1 (2017): 101-109. 